Santa Clara University

Scholar Commons

Electrical Engineering

School of Engineering

9-8-2008

\title{
Improved contact for thermal and electrical transport in carbon nanofiber interconnects
}

Tsutomu Saito

Toshishige Yamada

SantaClaraUniversity, tyamada@scu.edu

Drazen Fabris

Santa Clara University, dfabris@scu.edu

Hirohiko Kitsuki

Patrick Wilhite

Santa Clara University, pwilhite@scu.edu

See next page for additional authors

Follow this and additional works at: https://scholarcommons.scu.edu/elec

\section{Recommended Citation}

T. Saito, T. Yamada, D. Fabris, H. Kitsuki, P. Wilhite, M. Suzuki, and C.Y. Yang, "Improved contact for thermal and electrical transport in carbon nanofiber interconnects," Applied Physics Letters 93, 102108 (3 pp) (2008). https://doi.org/10.1063/1.2979710

Copyright (C) 2007 American Institute of Physics Publishing. Reprinted with permission.

This Article is brought to you for free and open access by the School of Engineering at Scholar Commons. It has been accepted for inclusion in Electrical Engineering by an authorized administrator of Scholar Commons. For more information, please contact rscroggin@scu.edu. 
Authors

Tsutomu Saito, Toshishige Yamada, Drazen Fabris, Hirohiko Kitsuki, Patrick Wilhite, Makoto Suzuki, and Cary Y. Yang 


\title{
Improved contact for thermal and electrical transport in carbon nanofiber interconnects
}

\author{
Tsutomu Saito, ${ }^{\text {a) }}$ Toshishige Yamada, Drazen Fabris, Hirohiko Kitsuki, Patrick Wilhite, \\ Makoto Suzuki, and Cary Y. Yang \\ Center for Nanostructures, Santa Clara University, Santa Clara, California 95053, USA
}

(Received 22 July 2008; accepted 19 August 2008; published online 10 September 2008)

\begin{abstract}
We study the performance and reliability of carbon nanofiber (CNF) interconnects under high-current stress by examining CNF breakdown for four test configurations, suspended/supported with/without tungsten deposition. The use of $\mathrm{W}$ is to improve the CNF-electrode contact. The supported cases show a larger current density just before breakdown than the suspended ones, suggesting an effective heat dissipation to the substrate. The W-deposited contacts reduce the initial total resistance from megaohm range without $\mathrm{W}$ to kilo-ohms. High-current stress does not change the total resistance of the test structures with $\mathrm{W}$ unlike those without $\mathrm{W}$ deposition. (C) 2008 American Institute of Physics. [DOI: 10.1063/1.2979710]
\end{abstract}

For carbon nanotubes ${ }^{1,2}$ (CNTs) or carbon nanofibers ${ }^{3,4}$ (CNFs) to be implemented as next-generation interconnect materials, ${ }^{5}$ their performance and reliability properties must be studied and well understood. In recent studies of thermal and electrical transports in CNT (Refs. 6-12) and CNF, ${ }^{13}$ it was shown that their breakdown is closely related to Joule heating. In our early work, it was observed that the maximum current density $J_{\max }$ is inversely proportional to the CNF length $L$ for suspended CNFs, which was explained with a heat transport model. ${ }^{14}$ During the experiment, thermal and electrical transports between the CNF and metal electrodes was improved by applying high dc to the system (current stressing), ${ }^{15}$ and the extent to which current stressing affects the CNF-electrode contact was analyzed. ${ }^{14}$ Here we report a systematic study of thermal and electrical transport measurements by comparing contacts with and without tungsten deposition on CNF either supported on substrate or suspended between a pair of electrodes, as shown in Fig. 1. These are (1) CNF suspended between two prepatterned planar electrodes on a $\mathrm{SiO}_{2} / \mathrm{Si}$ substrate, (2) CNF resting on a $\mathrm{SiO}_{2} / \mathrm{Si}$ substrate between two prepatterned electrodes, (3) CNF suspended between two prepatterned electrodes with $\mathrm{W}$ deposited on top of the CNF/electrode contact, and (4) CNF supported between two prepatterned electrodes with $\mathrm{W}$ deposited on top of the CNF/electrode contact.

CNFs used in these measurements have a cup-shaped morphology ${ }^{16}$ and were grown using plasma-enhanced chemical vapor deposition technique with Ni catalyst. ${ }^{17}$ The CNFs were removed from the substrate and immersed in isopropyl alcohol. To separate CNFs individually, the solution was sonicated for about $15 \mathrm{~min}$ before drop-cast onto a substrate of prepatterned $\mathrm{Au}$ electrodes on an oxidized silicon wafer. For configurations (3) and (4), W was deposited to improve the contact between the CNFs and metal electrodes using a focused ion beam system with source gas $\mathrm{W}(\mathrm{CO})_{6}$. W was deposited on each end of CNF such that the coverage area was approximately $1.2 \times 1.2 \mu \mathrm{m}^{2}$, and the $\mathrm{CNF}$ was sandwiched between the $\mathrm{Au}$ electrode and

\footnotetext{
a) Author to whom correspondence should be addressed. Electronic mail: tsaito1@scu.edu.
}

deposited W. The ion beam condition had to be optimized so as not to damage the CNF structure, and the ion beam irradiation to the bridged portion of CNF must be avoided. ${ }^{18}$ Electrical measurement was then performed to examine transport prior to breakdown from current stressing. To obtain the initial electrical characteristics, the current-voltage $(I-V)$ curve was measured in a small voltage range (a few millivolts) around $V=0$. Then, a constant current was applied

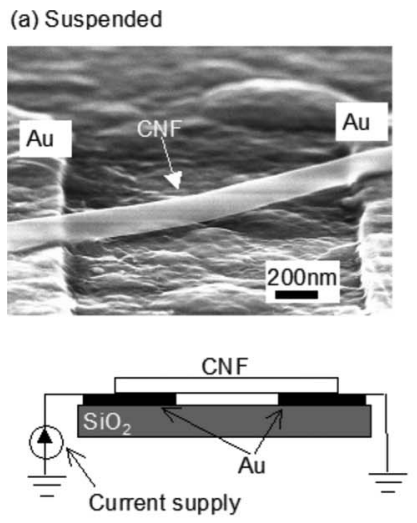

(c) Suspended with W deposition
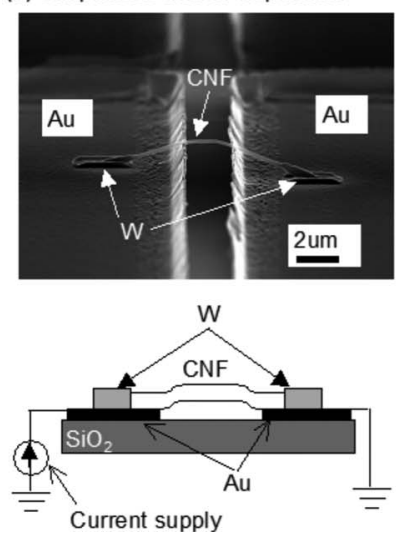

(b) Supported

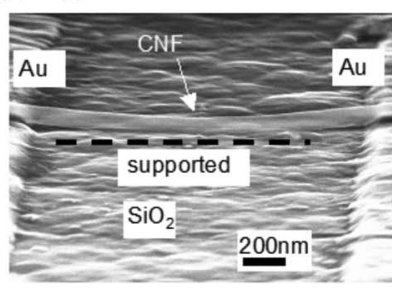

(d) Supported with W deposition
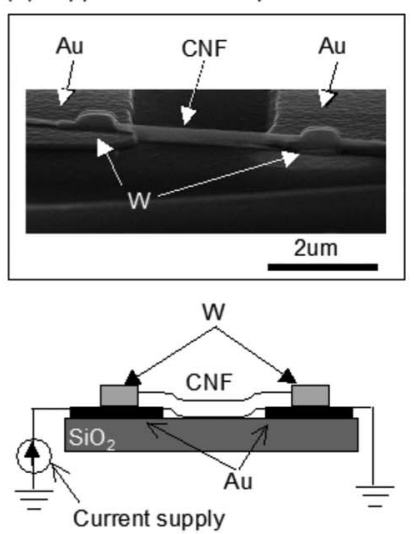

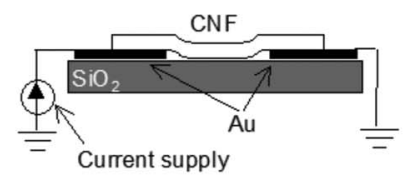

FIG. 1. Current-stressing experiments: (a) CNF suspended on gold electrodes; (b) CNF supported by $\mathrm{SiO}_{2}$ substrate; (c) CNF suspended over $\mathrm{SiO}_{2}$ substrate with W deposited on contacts; and (d) CNF supported by $\mathrm{SiO}_{2}$ substrate with $\mathrm{W}$ deposited on contacts. Upper figures show SEM image of a $\mathrm{CNF}$ device at $75^{\circ}$ tilted-angle view, and lower figures illustrate schematic of electrical measurement setup for current-stressing experiments. 

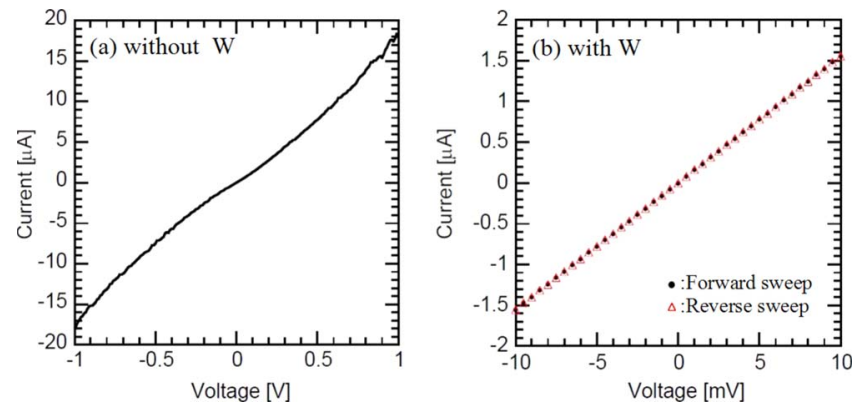

FIG. 2. (Color online) (a) Typical $I-V$ characteristics with configuration (1) measured before high-current stress. (b) Typical $I$-V characteristics with configuration (4) measured before high-current stress.

to the device for 3 min. Finally, the $I-V$ curve was remeasured under the same bias condition and the two results were compared. The current-stressing cycle was repeated with a new larger stressing current until breakdown occurred. Here we present results for 36 devices with CNFs ranging from 100 to $200 \mathrm{~nm}$ in diameter and from 1.5 to $8.6 \mu \mathrm{m}$ in length.

To minimize contact resistance between the CNF and metal electrodes for practical interconnect applications, configurations (3) and (4) with improved contact are studied. In these configurations, the region where the CNF is in contact with the Au electrode is fully covered with deposited W. Contacts fabricated with this process have a typical resistance in the kilo-ohm range, which is one to three orders of magnitude smaller than those for configurations (1) and (2).

The test structure is examined using scanning electron microscopy (SEM) to ensure that no structural damage was created by $\mathrm{W}$ deposition. By comparing the SEM images before and after $\mathrm{W}$ deposition, no difference is observed in the CNF. The $I-V$ curve for configuration (1) shown in Fig. 2(a) is nonlinear and noisy due to poor contact between $\mathrm{CNF}$ and electrodes. The resistance at $V=0$ is $87.6 \mathrm{k} \Omega$. This nonlinearity suggests tunneling transport between $\mathrm{CNF}$ and electrodes ${ }^{19}$ Fig. 2(b) shows the $I-V$ behavior for configuration (3) with $\mathrm{W}$ contact. The resistance is $6.4 \mathrm{k} \Omega$, typical of devices with $\mathrm{W}$ deposition. Solid circles show measurement results from forward voltage sweep, and open triangles show reverse voltage sweep. These two curves overlap quite well and show no hysteresis. Moreover, the $I-V$ curve is linear and free from noise. Comparison of results from these two configurations clearly shows that $\mathrm{W}$ deposition on the contacts improves the contact resistance between CNF and electrodes.

Results for devices subject to current stressing are shown in Fig. 3. For configuration (1) (solid circles), our previous work demonstrated that breakdown always occurs at or near the middle of the nanofiber. ${ }^{14}$ Furthermore, the proposed thermal transport model predicts that the highest temperature is reached in the middle of the $\mathrm{CNF},{ }^{14}$ while confirming that the maximum current density $J_{\max }$ just before breakdown is inversely proportional to the length $L$ for the suspended CNF, as illustrated by the straight line in Fig. 3. For configuration (2) (open circles), the $\mathrm{CNF}$ region contacted to the $\mathrm{SiO}_{2} / \mathrm{Si}$ substrate can dissipate heat more effectively from the nanofiber to the substrate where the two are in contact. Thus the supported CNFs in configuration (2) show significantly higher maximum current density in comparison to the suspended ones in configuration (1).

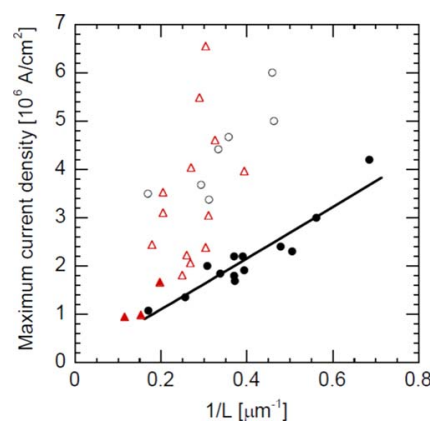

FIG. 3. (Color online) Maximum current density as a function of reciprocal CNF length for 36 devices. Solid circles indicate the results for configuration (1), open circles for configuration (2), solid triangles for configuration (3), and open triangles for configuration (4). The solid line shows a linear fit for suspended CNFs, as predicted by our heat transport model (Ref. 14).

For configuration (3), suspended CNF with W contact (solid triangles in Fig. 3), the maximum current density as a function of $1 / L$ behaves the same as that for configuration (1) regardless of the contact improvement. The fact that both suspended cases can be described by the same linear relationship further confirms the validity of the thermal transport model, ${ }^{14}$ which assumes dissipation mainly through the contacts. This finding suggests that the reduction in contact electrical resistance from configurations (1) to (3) due to deposited $\mathrm{W}$ does not seem to alter the overall transport of Joule heat for suspended CNFs. For configuration (4), supported $\mathrm{CNF}$ with $\mathrm{W}$ contact (open triangles), the maximum current density is significantly and consistently higher compared to the suspended cases of configurations (1) and (3). These results demonstrate that for both supported CNF cases, heat dissipation in the CNF segments in contact with the substrate dominates the transport and leads to higher maximum current density.

For W-deposited cases, current stressing has little effect on the total resistance. Figure 4 shows the total resistance measured at the end of each current-stressing cycle as a function of stress current with and without $\mathrm{W}$ deposition. Without W [Fig. 4(a)], the total resistance decreases as higher stressing current is applied. Initially, each device has a different CNF resistance due to variations in diameter and/or length as well as contact resistance between the CNF and electrodes. For that reason, the initial total resistance varies between 0.1 and $10 \mathrm{M} \Omega$. After successive current stressing cycles, the resistance variation starts to diminish, reaching a total resistance of several kilo-ohms just before breakdown. On the
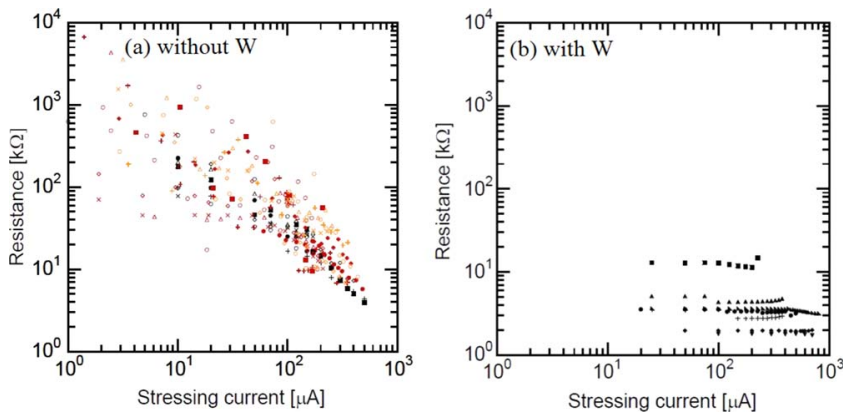

FIG. 4. (Color online) (a) Resistance of CNF devices without W deposition as a function of annealing current after each annealing cycle. (b) Resistance of CNF devices with $\mathrm{W}$ deposition as a function of annealing current after each annealing cycle. 
other hand, devices with $\mathrm{W}$ contact [Fig. 4(b)] show very little effect from current stressing, with total resistance decreasing by at most $20 \%$. Our results suggest that current stressing mainly improves the contact, and if the contact is already electrically optimum at the outset, there is a little room for further improvement from current stressing. Also our results infer that CNF bulk resistance is not affected by current stressing except at breakdown. At each currentstressing cycle, while Joule heat is generated within the nanofiber structure, the bulk CNF resistance remains virtually unchanged, consistent with the metallic characteristics of $\mathrm{CNF}$.

Suspended and supported CNFs with and without W deposition at the CNF/electrode contacts are studied experimentally. For suspended CNFs, the maximum current density $J_{\max }$ is inversely proportional to its length with and without the presence of W. For supported CNFs, heat dissipation to the substrate is significant, and $J_{\max }$ is significantly higher than that for suspended CNFs. Current stressing is shown to improve mainly the properties of the CNF/electrode contact. Initial resistance of W-deposited contact is typically a few kilo-ohms and remains about the same throughout current stressing until breakdown. This study provides the experimental basis for further elucidating the electrical and thermal transport and breakdown mechanisms in CNF interconnects.

We are grateful to Kevin Mcilwrath and Xiao Feng Zhang of Hitachi High-Technologies America for their expert technical support. This work was supported by the United States Army Space and Missile Defense Command (SMDC) and carries Distribution Statement A, approved for public release, distribution unlimited.
${ }^{1}$ J. Li, Q. Ye, A. M. Cassell, H. T. Ng, R. Stevens, J. Han, and M. Meyyappan, Appl. Phys. Lett. 82, 2491 (2003).

${ }^{2}$ M. Nihei, A. Kawabata, D. Kondo, M. Horibe, S. Sato, and Y. Awano, Jpn. J. Appl. Phys., Part 1 44, 1626 (2005).

${ }^{3}$ A. V. Melechko, V. I. Merkulov, T. E. McKnight, M. A. Guillorn, K. L. Klein, D. H. Lowndes, and M. L. Simpson, J. Appl. Phys. 97, 041301 (2005)

${ }^{4}$ Q. Ngo, A. M. Cassell, A. J. Austin, J. Li, S. Krishnan, M. Meyyappan, and C. Y. Yang, IEEE Electron Device Lett. 27, 221 (2006).

${ }^{5}$ W. Hoenlein, F. Kreupl, G. S. Duesberg, A. P. Graham, M. Liebau, R. V. Seidel, and E. Unger, IEEE Trans. Compon. Packag. Technol. 27, 629 (2004).

${ }^{6}$ P. G. Collins, M. Hersam, M. Arnold, R. Martel, and Ph. Avouris, Phys. Rev. Lett. 86, 3128 (2001).

${ }^{7}$ B. Bourlon, D. C. Glattli, B. Plaçais, J. M. Berroir, C. Miko, L. Forró, and A. Bachtold, Phys. Rev. Lett. 92, 026804 (2004).

${ }^{8}$ A. Javey, J. Guo, M. Paulsson, Q. Wang, D. Mann, M. Lundstrom, and H. Dai, Phys. Rev. Lett. 92, 106804 (2004).

${ }^{9}$ J. Y. Huang, S. Chen, S. H. Jo, Z. Wang, D. X. Han, G. Chen, M. S. Dresselhaus, and Z. F. Ren, Phys. Rev. Lett. 94, 236802 (2005).

${ }^{10}$ T. D. Yuzvinsky, W. Mickelson, S. Aloni, S. L. Konsek, A. M. Fennimore, G. E. Begtrup, A. Kis, B. C. Regan, and A. Zettl, Appl. Phys. Lett. 87, 083103 (2005).

${ }^{11}$ M. A. Kuroda, A. Cangellaris, and J.-P. Leburton, Phys. Rev. Lett. 95, 266803 (2005).

${ }^{12}$ E. Pop, D. A. Mann, J. Cao, K. E. Goodson, and H. Dai, J. Appl. Phys. 101, 093710 (2007).

${ }^{13}$ M. Suzuki, Y. Ominami, Q. Ngo, C. Y. Yang, J. Li, and A. M. Cassell, J. Appl. Phys. 101, 114307 (2007).

${ }^{14}$ H. Kitsuki, T. Yamada, D. Fabris, J. R. Jameson, P. Wilhite, M. Suzuki, and C. Y. Yang, Appl. Phys. Lett. 92, 173110 (2008).

${ }^{15}$ J.-O. Lee, C. Park, J.-J. Kim, J. Kim, J. W. Park, and K.-H. Yoo, J. Phys. D 33, 1953 (2000).

${ }^{16}$ Y. Ominami, Q. Ngo, M. Suzuki, A. J. Austin, and C. Y. Yang, Appl. Phys. Lett. 89, 263114 (2006).

${ }^{17}$ B. A. Cruden, A. M. Cassell, Q. Ye, and M. Meyyappan, J. Appl. Phys. 94, 4070 (2003).

${ }^{18}$ Y. J. Jun, Y. Homma, R. Vajtai, Y. Kobayashi, T. Ogino, and P. M. Ajayan, Nano Lett. 4, 1109 (2004).

${ }^{19}$ T. Yamada, Appl. Phys. Lett. 78, 1739 (2001). 